\title{
EVALUACIÓN SENSORIAL DE UN EMULSIONANTE EN EL PROCESAMIENTO DE CREMA DE ACEITUNA NEGRA
}

Responsable: Msc. Marcial Alfredo Castillo Cohaila

\section{RESUMEN}

Se utilizaron aceitunas negras fermentadas para la elaboración de crema de aceitunas, preparadas con diferentes cantidades de emulsionante lecitina, determinándose mediante evaluación sensorial que las cremas de aceitunas negras con $1 \%$ de lecitina y sin lecitina no tienen diferencia para una significancia del $5 \%$ por el método de datos pareados.

\begin{abstract}
We used fermented black olives, for the manufacture of olive cream, prepared with different amounts of emulsifier lecithin, determined by sensory evaluation that the creams black olives with $1 \%$ of lecithin and without lecithin, have no difference, for a significance of $5 \%$, By the method of paired data.
\end{abstract}

\section{INTRODUCCIÓN}

Según Ranken (1993), básicamente las cremas son una emulsión viscosa de aceite en agua con yema de huevo como agente emulgente primario o único, y que contienen un agente acidificante que normalmente es vinagre. Otros ingredientes pueden ser azúcar, mostaza, sal, espesantes, especias, aromatizantes y colorantes.

La mayoría de las cremas contienen glóbulos de aceite con un diámetro de alrededor de 5 micrómetros y se encuentran dispersos en toda la fase acuosa.

La fosfatidilcolina (también llamada lecitina) junto con las sales biliares ayudan a la solubilización de los ácidos biliares en la bilis.

La fosfatidilcolina contiene mayoritariamente ácido palmítico o ácido esteárico en la posición del C-1 y principalmente los ácidos grasos insaturados de 18 carbonos, oleico, linoléico o linolénico en la posición de C-2. No se ha limitado la ingestión diaria admisible. La lecitina es un componente esencial de los jugos biliares, que aportan diariamente al intestino de 10 a 12 gramos, mucho mas que el que procede de la dieta, que es solo de uno o dos gramos por dia, contando tanto el propio de los alimentos como el utilizado como aditivo. En el intestino facilita la absorción de las otras grasas, actuando cömo emulsionante de la misma forma que lo hace en los alimentos. Es considerado como un aditivo totalmente seguro, incluso por aquellas personas fanáticas de los alimentos naturales.

Los objetivos del presente trabajo de investigación son los siguientes:

\section{Objetivo principal}

Determinar el efecto de la incorporación de un agente emulsionante con base de lecitina en la elaboración de crema de aceituna negra

\section{Objetivos específicos}

Determinar la cantidad de agente emulsionante óptima para la estabilidad de la crema de aceituna negra.

Determinar si la incorporación del agente emulsionante afecta el sabor u olor de la crema de aceituna negra.

Determinar la mejor alternativa con base de lecitina, para la elaboración de crema de aceituna negra.

\section{MATERIAL Y MÉTODOS}

El proyecto de investigación se desarrolló en los ambientes de la Facultad de Ingeniería en Industrias Alimentarias.

\section{Recursos Materiales:}

Los recursos materiales utilizados fueron:

- Aceitunas negras: 40 kilogramos

- Depósito plástico (30 litros) para fermentación de aceitunas: 02 unidades

- Sal, ácido acético, sorbato de potasio, agua potable.

- Huevos frescos

- Lecitina de soya.

- Material de laboratorio: densímetro Baumé, probeta, pipetas, balanza analítica, $\mathrm{pH}$-metro, etc. 
- 01 computadora Pentium IV

- Papelbond

2. Panelistas:Los panelistas o jueces para la realización de la evaluación sensorial serán en total 8 , los cuales tendrán un concepto general del propósito de la prueba, asi como las caracteristicas del producto en evaluación.

3.Metodología: El proyecto se desarrolló por la metodologia que se observa en la Figura 1: aceitunas negras fermentadas, crema de aceituna negra con lecitina a concentraciones de $0,05 \%, 0,1 \%, 0,15 \%$ y $0,2 \%$, mejor crema de aceituna negra con lecitina, crema de aceituna negra sin lecitina, comparación de las cremas de aceituna negra con y sin lecitina, resultados $y$ conclusiones.

Para la elección de la mejor crema de aceituna con lecitina a concentraciones de $0,05 \%, 0,1 \%, 0,15 \%$ y $0,2 \%$, se realizó una prueba sencilla de preferencia, considerándose para la comparación, aquella muestra que obtuvo el más alto puntaje.

4.Procedimiento:Para la elaboración de la crema de aceituna, en la Figura 2, podemos observar el flujo de operaciones de la crema de aceitunas negras: aceitunas negras, fermentación, deshuesado, pulpeado, tratamiento térmico, enfriado, mezclado, crema de aceitunas negras.

5.Para la evaluación sensorial:Previamente, para el desarrollo del diseño experimental entre las muestras con emulsionante, se escogió la muestra con mejor puntaje, por medio de la prueba de Kramer, con una significancia de $5 \%$.

\section{RESULTADOS}

Cantidad de emulsionante en la crema de aceitunas negras con lecitina.

En el Cuadro 1 se observan los resultados de la evaluación sensorial en la crema de aceituna negra con el emulsionante. Nos muestra los puntajes para cada tratamiento con emulsionante. Los puntajes utilizados fueron valores enteros del 1 al 4 , observándose en el Cuadro que la muestra $B$ registra mayores puntajes de parte de los jueces.

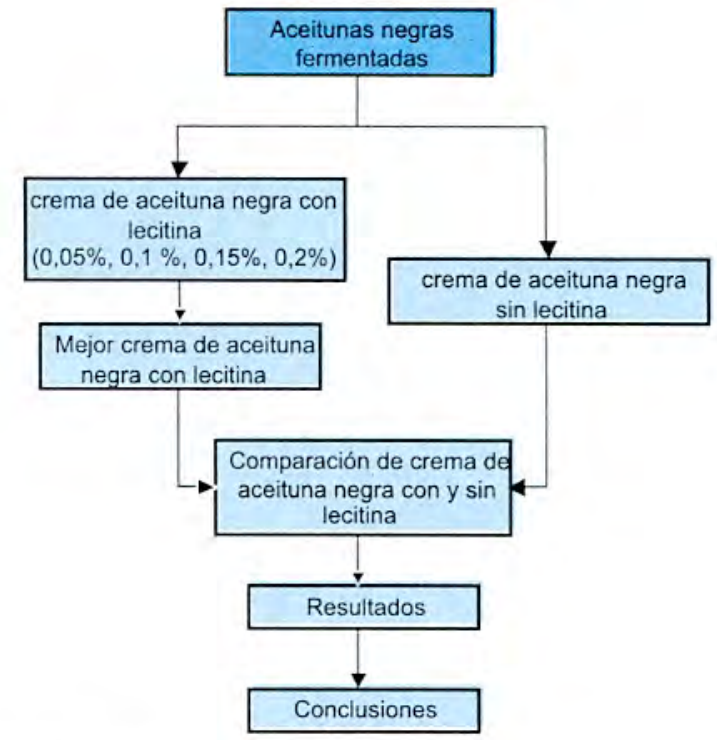

Fig 1: Metodologia de la investigación

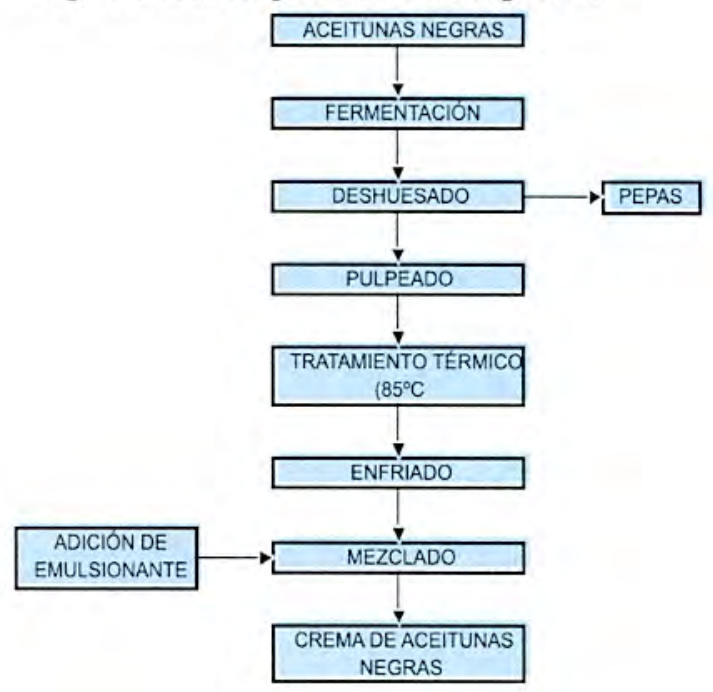

Fig 2: Proceso de elaboración de crema de aceituna negra.

Cuadro 01: Puntaje según $\%$ de lecitina

\begin{tabular}{|c|c|c|c|c|}
\hline \multirow{2}{*}{ Juez } & \multicolumn{4}{|c|}{ Puntaje del tratamiento } \\
\cline { 2 - 5 } & A & B & C & D \\
\cline { 2 - 5 } & $\mathbf{0 , 0 5}$ & $\mathbf{0 , 1 0} \%$ & $\mathbf{0 , 1 5} \%$ & $\mathbf{0 , 2 0 \%}$ \\
\hline $\mathbf{1}$ & 3 & 4 & 2 & 1 \\
\hline 2 & 1 & 4 & 3 & 2 \\
\hline 3 & 4 & 3 & 2 & 1 \\
\hline 4 & 2 & 4 & 3 & 1 \\
\hline 5 & 3 & 4 & 1 & 2 \\
\hline 6 & 2 & 4 & 3 & 1 \\
\hline
\end{tabular}

1. Análisis estadístico:Para determinar la mejor muestra de crema de aceituna negra con emulsionante.

Se utilizó la prueba de Kramer, para lo cual 
se ordenaron los tratamientos según orden creciente, tal como se observa en el Cuadro 2.

Cuadro 02: Ordenación creciente por puntaje de los tratamientos con emulsionante

\begin{tabular}{|c|c|c|c|c|}
\hline \multirow{2}{*}{ Juez } & \multicolumn{4}{|c|}{ Puntaje del tratamiento } \\
\cline { 2 - 5 } & D & C & A & B \\
\cline { 2 - 5 } & $\mathbf{0 , 2 0} \%$ & $\mathbf{0 , 1 5} \%$ & $\mathbf{0 , 0 5} \%$ & $\mathbf{0 , 1 0} \%$ \\
\hline $\mathbf{1}$ & 1 & 2 & 3 & 4 \\
\hline $\mathbf{2}$ & 2 & 3 & 1 & 4 \\
\hline $\mathbf{3}$ & 1 & 2 & 4 & 3 \\
\hline $\mathbf{4}$ & 1 & 3 & 2 & 4 \\
\hline $\mathbf{5}$ & 2 & 1 & 3 & 4 \\
\hline $\mathbf{6}$ & 1 & 3 & 2 & 4 \\
\hline Total & 8 & 14 & 15 & 23 \\
\hline
\end{tabular}

Fuente. Elaboración propia.

En la Figura 3 se observa el diagrama de Kramer, obteniéndose el rango entre 9 y 21 con un nivel de significancia del $5 \%$, con lo que podemos afirmar que las muestras con $0,2 \%$ de emulsionante son las que menos aceptación presentaron; las muestras con $0,05 \%$ y $0,15 \%$ de emulsionante presentaron similar aceptación, mientras que las muestras con $0,1 \%$ de emulsionante fueron las más aceptadas; por lo tanto, son las que se utilizaron para realizar la comparación con las muestras sin emulsionante.

\begin{tabular}{c|cc} 
& $9-21$ & \\
\hline a & b & C \\
\hline D & C A & B \\
\hline
\end{tabular}

Para analizar las cremas de aceituna negra con y sin emulsionante, se evaluó mediante el análisis estadístico de datos pareados, el color, olor, sabor, textura y aspecto general de la crema elaborada.

Respecto al color, olor, sabor, textura y aspecto general de las cremas de aceituna, se estableció que no existe diferencia significativa entre los dos tratamientos; por lo tanto, las aceitunas sin emulsionante y con emulsionante son estadísticamente similares.

\section{DISCUSION}

Para mantener la estabilidad de la emulsión de las cremas, Ranken (1993), explica que esto se logra con no menos de $1,35 \%$ de sólidos de yema de huevo, lo que ayuda a conseguir la consistencia requerida y a eliminar el riesgo de rotura de la emulsión durante el transporte y almacenamiento. Esta estabilidad se pudo comprobar en los tratamientos realizados en la crema de aceituna sin lecitina como agente emulsionante.

Sin embargo, en lo referente a los tratamientos con lecitina como agente emulsionante, en los cuales se encontró resultados similares en cuanto a la estabilidad de la crema de aceituna negra con la crema de aceituna negra preparada sin lecitina, reafirma lo indicado por Ranken (1993), que sostiene que es factible el uso de otros emulsionantes, aunque él hace referencia a la incorporación de un emulgente secundario; que en caso de utilizar como emulgente primario la yema de huevo, ya no sería necesario el emulgente secundario.

Asimismo, Márquez et al. (2005) señalan que la desestabilización (separación gravitacional, floculación y coalescencia) de las emulsiones aceite en agua se pueden minimizar con la reducción del tamaño de la gota, por lo cual será importante analizar el efecto de la agitación controlada sobre la estabilidad frente al mecanismo de la coalescencia.

\section{CONCLUSIONES}

1.No existe diferencia entre crema de aceituna negra emulsificada con lecitina al $0,1 \%$ y crema de aceituna negra sin lecitina.

2.La estabilidad de la crema de aceituna negra sin lecitina es similar que cuando se utiliza lecitina en $0,1 \%$ de concentración.

3.La adición de lecitina en dosis de $0,1 \%$ de concentración no afecta ni el sabor ni olor de la crema de aceituna negra.La mejor alternativa para emulsificar la crema de aceituna negra con lecitina es de $0,1 \%$ de concentración.

\section{RECOMENDACIONES}

Evaluar el emulsionante lecitinam combinando con otros emulsionantes.

Realizar evaluaciones utilizando otros u otros agente emulsionantes.

Realizar el pulpeado de las aceitunas, en un molino coloidal y establecer si esta operación afecta el resultado de las acciones del emulsionante.

\section{BIBLIOGRAFÍA}

Anzaldúa Morales, A. (1994). La evaluación sensorial de los alimentos en la teoría y la 
práctica. Editorial Acribia, Zaragoza, España.

Márquez A., L.; Palazolo G., G., Wagner J., R. (2005). Grasas y Aceites. Volumen 56. Fascículo 2. Págs. 89 - 95.

Beriain-Apesteguia, M.; Alfonso-Ruiz, L. y Gorraiz-Olangua, C. (2001). "Diseño y análisis estadístico de los experimentos sensoriales. 142:180." En: Análisis sensorial de alimentos. métodos y aplicaciones. Ibáñez, F. C.; Barcina, Y.; Editores. Springer- Verlag Ibérica. Barcelona.

Espinoza Atencia, E. (2003). Evaluación sensorial de los alimentos. Tacna-Perú.

Fisher, C. y Scott, T. R. (2000). Flavores de los alimentos. Biología y química. Editorial Acribia, Zaragoza, España.

Fortin, J. y Desplancke, C. (2001). Guía de selección y entrenamiento de un panel de catadores. Editorial Acribia, Zaragoza, España.

Issanchou, S.; Lesschaeve I. \& Köster E.P. (1995). "Screening individual ability to perform descriptive analysis of food products: basic statements and application to a camembert cheese descriptive panel". Journal of Sensory Studies, 10, 349-368.

Ranken, M.D. (1993). Manual de industrias de los alimentos. 2da. edición. Editorial Acribia S.A., Zaragoza, España, 332, 437.

Rosenthal, A. 2001. Textura de los alimentos. Medida y percepción. Editorial Acribia. Zaragoza España.

Salamanca, G. G. (s.a.). Criterios relativos al análisis sensorial de mieles. Departamento de Química. Ibagué, Tolima, Colombia 